\title{
SKIN TESTING IN CLINICAL DIAGNOSIS
}

\author{
Major P. D. MEERS* \\ M.D., M.C.Path., Dip.Bact., R.A.M.C. \\ Royal Army Medical College, Millbank
}

SkIN testing is a direct means of applying the immunological response of the body to clinical diagnosis, by observing the result of the introduction into the skin of a test antigen. Because a clear knowledge of immunology is so important to a proper understanding of the use of these antigens, the basic outlines of the science as applied to man are briefly recapitulated.

It is 'customary to divide the body's immunological response to antigenic stimulus into two broad classes; first, that involving classical circulating antibody, and second, the hypersensitive or allergic state. The latter is again divided into immediate and delayed hypersensitivity. Classical antibody is that which may be made to react directly with its antigen in: some readily observable way in the laboratory. This is the antibody of the Widal, Weil-Felix or Brucella agglutination tests and is illustrated by the Schick and Dick tests. Immediate hypersensitivity occurs in the presence of circulating antibody, which may be of the classical type, or may be of the incomplete (univalent, non-precipitating) or reagin type. The incomplete and reagin type antibodies are not demonstrable directly in the laboratory, but require some additional indicator to detect that an antigenantibody reaction has taken place in vitro, or they may be demonstrated by transferring serum, and with it the hypersensitive reactivity, from a sufferer to a "normal" person. Hay fever, asthma, some drug and food idiosyncrasies and urticaria are examples of immediate hypersensitivity. One of the characteristics of this type of reaction, from which it takes its name, is the speed with which the reaction occurs-usually within minutes, and certainly within the first 24 hours of the introduction of the antigen into the sensitised individual.

The delayed hypersensitive state is completely different. In eliciting this response, a delay between the introduction of the antigen and the appearance of the reaction of at least several hours occurs, the reaction reaching a maximum in 24 to 72 hours, and persisting for several days. This type of reaction is typified by the tuberculin test,' but is also seen in many other infections (being sometimes called the "allergy of infection "), and it is probably the cause of what has been called the "reaction of immunity " following smallpox vaccination. It is thought to be concerned in homograft rejection, and contact dermatitis (" contact allergy ") also falls into this group. In delayed hypersensitivity no circulating antibody is demonstrable by any in vitro means, or by the transfer of serum from a hypersensitive to a normal subject. Passive transfer of this type of reactivity is only possible by the infusion of cell suspensions (lymphocytes especially) from the one to the other.

It should be realised that any given individual, when faced by a new antigen, may produce none of these three basic responses, or one, two, or under certain circumstances all three types of reacitivity, sequentially or concurrently. Also, any one antigen may produce quite different spectra of reactivity in different individuals. It is important to 
remember that the presence of delayed hypersensitivity to an infectious agent does not imply de facto immunity in the sense of an increased resistance to infection with that agent, though the two factors often go hand in hand.

In diagnostic skin testing, the immunological facts set out above are put to work in one of two ways. In testing for classical immunity, a toxic extract of the disease-producing agent is used, which, in non-immune persons, produces a local inflammatory reaction at the site of inoculation. In an immune individual however, circulating antibody neutralises the toxin, and the production of the reaction is inhibited. When hypersensitivity is being looked for, a non-toxic antigen is prepared from the agent, which in non-hypersensitive persons causes no reaction, but in the hypersensitive causes an inflammatory reaction of the immediate or of the delayed type. The immediate reaction is of the weal and flare sort, coming on within minutes of the inoculation. This appears to be due to the release of histamine-like substances by cells damaged as a result of antigen-antibody union. The delayed reaction consists of erythema accompanied by induration, the latter being due to cellular infiltration. This' appears slowly, reaching a maximum in 24 to 48 , or occasionally 72 hours, remaining obvious for several more days. It is usually stressed that the induration is the essential part of this reaction, and in measuring such a reaction to decide on its significance, erythema is ignored. There remain two diagnostic skin reactions which do not appear to fall into any of the three immunological groups described. These are the late (Mitsuda) reaction to lepromin in tuberculoid leprosy, and the reaction to the Kveim antigen in sarcoidosis. A positive reaction in both these tests is denoted by an area of nodular infiltration, reaching its maximum in three to five weeks. In the Kveim test, a biopsy of the inoculated area should be examined in every case, even those appearing negative macroscopically.

The lepromin test is unique in that a tuberculin-type delayed reaction, called (unfortunately) the immediate or Fernandez reaction maybe followed some weeks later by the delayed or. Mitsuda reaction. Both reactions seem to indicate the degree of bodily reactivity to Mycobacterium leprae, in those sensitised by infection with it. It follows, then, that positive Fernandez and Mitsuda reactions occur with greater frequency in cases of dimorphic leprosy which have dominant tuberculoid lesions, and both are characteristically positive in "pure" tuberculoid leprosy. Just as a patient with miliary tuberculosis may revert to a negative tuberculin test, so a patient with florid lepromatous leprosy will tend to have a negative lepromin tests of both types.

The antigens themselves are, with exceptions in two well defined groups; extracts of the infective agent the diagnosis of the disease due to which is being sought. The exceptions are the antigens used in the determination of the "allergens" active in hay fever, asthma, etc., and those employed in patch-testing for the precipitating factor in contact dermatitis. These are extracts of pollens, danders, dusts, etc., on the one hand, and a variety of chemicals on the other. For all the rest, the infective agent is grown, either on bacteriological media or in eggs in the laboratory, or is obtained as the result of a natural, or artifically produced infection in an animal, or as the result of natural infection in man. Where it can be grown in vitro, or, as with an intestinal or tissue parasite, may be obtained otherwise in a high state of purity, an antigen can be prepared with little or no contaminating non-specific material. With tuberculin, this process has been carried one stage further, and the active principle has been extracted from the mycobacterium, producing the "purified protein derivative" (PPD). This final step gives a product virtually free from material which might give a non-specific effect, and which produces 
reactions which are as highly reproducible from batch to batch as is possible for any skin testing antigen. All other skin test antigens contain more than one antigenic determinant derived from the infective agent. This is because all such agents are structurally complex, and each part of the structure is potentially antigenic. The relative amounts of these different antigens will inevitably vary from batch to batch of test antigen, and this may influence the comparability of tests done on different occasions. The tuberculins (other than PPD), and Schick and Dick test toxins are sufficiently defined to have a high reproducibility. For the rest, little completely valid attempt at comparative titration is, or indeed can be made. Apart from the multiple antigens which are derived from the infective agent itself, many skin test materials contain some residues from the medium in which the agent was grown, some of which may themselves be antigenic, and which may produce non-specific effects. This is particularly important in virus and some parasitic skin test antigens, where a growth of the inefective agent can only be obtained in the presence of living cells of embryonated eggs or of intact animals, and in which the test antigen cannot be freed from the large quantities of contaminating host material. In such tests, it is usual to employ a control inoculation duplicating the test in all respects, except that the test antigen itself is missing. By this means, false positive reactions due to such contaminating material may be detected. Finally three skin test antigens are prepared from húman material, carrying with them the theoretical risk of isoimmunisation. The first of these, lepromin, is prepared from human lepromatous material because Myco. leprae has not yet been grown in vitro. The second is cat scratch fever antigen. This disease is thought to be due to a virus, which as yet has not been isolated, so the only source of antigen is the pus from the buboes of human cases of the disease. The third is the Kveim antigen, prepared from human sarcoid material, the cause of the disease being unknown.

The methods used for the introduction of the antigen into the skin are multifarious, ranging from simple application to the unabraded skin, through varying methods of abrasion, scarification or single or multiple puncture (by spring-loaded Heaf gun in its most highly developed form), to intracutaneous injection.

The Table contains a list of diseases for which diagnostic skin test antigens have been prepared. The list is not exhaustive, but includes all the commonly used antigens, and a selection of others intended to give some impression of the range and diversity of the tests which have been used in the past, or are still in limited use.

A diagnostic test needs to be simple, sensitive, specific, safe, significant, reproducible and practicable. To the clinician, skin testing is simple and has the added attraction that the result is available to him rapidly, without intervention. Skin testing, with a good antigen, is a very sensitive method, though from what has been said above, variations in sensitivity from batch to batch or from maker to maker are bound to occur. The specificity of skin testing is, in theory, excellent, but in practice most antigens as prepared are so antigenically heterogenous that a degree of specificity is lost. This may not be of critical importance however, and the degree of specificity may be sufficient for most clinical purposes. Safety is a more difficult subject. The " lighting up " of tuberculous foci by the injudicious use of tuberculin is probably a thing of the past, but similar effects might occur should a large dose of an antigen be given unwittingly to a patient with delayed hypersensitivity to that antigen. This is so particularly as most antigens cannot be, or are not adequately standardised. At times, too, reactions of the immediate hypersensitivity type may be of frightening intensity Another factor which requires to be considered under this heading is that the introduction of an antigen into the body is, in 
effect, vaccinating the patient with that antigen. At best, this will make difficult or impossible any future interpretation of serological findings in relation to the patient's disease, also, the patient may be sensitised to some of the extraneous antigens present in the test dose, such as egg protein or horse serum. The significance, or interpretation of the result of a skin test is not straightforward. This may be illustrated by the fact that a positive Mantoux or Heaf test does not necessarily indicate active tubercle, a positive lepromin test may be found in persons who are not currently suffering from leprosy and a negative result obtained in some of those who are, and that a patient who has at one time suffered from lymphogranuloma venereum may retain a positive Frei test for life. The reproducibility of most skin testing is in some doubt, as has been discussed above. The practicability of the use of skin test antigens tends to be forgotten by clinicians. Any material for injection into man requires, very properly, to be made to the specifications of the Therapeutic Substances Act. To make it worth while, a demand for large quantities of a skin test antigen must exist to justify the expense of setting up a manufacturing system. Some antigens can be made relatively easily, but others are difficult to prepare, and in some cases there is considerable difficulty in obtaining the raw material from which they are made. From this it follows that relatively few skin test antigens are regularly available. Others may be prepared by an investigator for a particular project, and as a result any excess is obtainable for general use until the supply is exhausted. Some can only be made when suitable raw material is found, the Kveim antigen for the diagnosis of sarcoidosis being a good example of this class.

If the trouble is taken to apply each of the seven criteria for a diagnostic test cited above to each of the skin test antigens in common use, relatively few of them emerge with credit. The tuberculin, Schick and Dick tests are the only ones which do not have severe disadvantages. If attention is then turned to the availability of an alternative diagnostic technique which may be substituted for the skin test, and the same seven criteria applied to these, it is immediately apparent that in nearly every case a better alternative technique does exist. However, the tuberculin, Schick and Dick tests still come out as the favourities; to them must be added cat scratch fever; Kveim and lepromin skin tests for which no satisfactory serological alternatives are available, though biopsy techniques may be preferred to skin tests in these diseases.

It may be instructive to examine one such comparison in detail-the Frei test compared with the complement fixation test (CFT) for lymphogranuloma venereum (LGV). For simplicity the skin test is favoured. When the two are compared for sensitivity, what information there is suggests that the CFT is more characteristically positive as a result of infection than is the skin test. The specificity of the Frei test is often stated to be high, though when deliberately looked for, the test is positive is some cases of virus pneumonia, while a "Frei" antigen prepared from the psittacosis virus gives positive results in LGV. The CFT, as usually performed, also does not distinguish between psittacosis and LGV, though the differential diagnosis between these two conditions presents no clinical problem. The safety of the two procedures cannot be comparedin one a mixed antigenic "vaccine" is being inoculated into a patient, the antigen including egg protein, in the other the patient is exposed only to venepuncture. In the significance of the result of the test, the CFT scores heavily. A Frei test seems to remain positive for long periods after infection with the virus of LGV, so that a positive result only indicates a past or present infection with the virus of LGV, or indeed with the virus of psittacosis. The CFT, on the other hand, done with a pair of sera collected some time 
apart, will nearly always give a better idea of whether the infection is an old one, or is current. The CFT is much more reproducible than the skin test, because in each test set up, the antigen is tested against a stock reference immune serum. On balance, it will be readily appreciated that the complement fixation method for the diagnosis of LGV is to be preferred to the intradermal test.

\section{Summary}

The immunological reaction of the body to the introduction of antigen is discussed, in relation to the employment of skin test antigens. A representative collection of such antigens is listed, and their use is considered more fully, in relation to a set of criteria for a diagnostic technique. The conclusion is drawn that, with the definite exceptions of the tuberculin, Schick and Dick tests, and the possible exceptions of the lepromin, Kveim, and cat scratch fever skin test antigens, little or no justification exists for their employment in man under normail circumstances.

TABLE A list of some diseases for the diagnosis of which skin test antigens have been prepared, classified according to the mechanism of the test.

\begin{tabular}{c} 
Mechanism of Test \\
\hline Tests depending on classical antibody \\
Tests depending on immediate hypersensitivity \\
\hline
\end{tabular}

Tests depending on delayed hypersénsitivity

\section{DISEASE}

Diphtheria (Schick test)

Scarlet fever (Dick test)

Parasitic infections

Ascariasis

Cysticercosis

Echinococcosis (Casoni antigen)

Filariasis

Schistosomiasis

Strongyloidiasis

Trichinosis

Pneumococcal pneumonia

\section{Bacterial infections}

Tests for "allergens" in asthma, hay fever, etc.

Brucellosis (brucellin)

Chancroid

Coccidioidomycosis

Glanders

Histoplasmosis

Leprosy (lepromin-Fernandez reaction)

Pertussis

Tuberculosis (tuberculin)

Tularaemia

Virus and rickettsial infections

Lymphogranuloma venereum (Frei antigen)

Mumps

Vaccinia/smallpox

Diseases of uncertain aetiology

Cat scratch fever

Tests for sensitising antigens in contact dermatitis.

Leprosy (lepromin-Mitsuda reaction)

Sarcoidosis (Kveim antigen) 